\title{
Construction and application of a built-in dual luciferase reporter for microRNA functional analysis
}

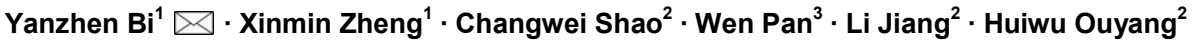 \\ 1 Hubei Key Laboratory of Animal Embryo Engineering and Molecular Breeding, Institute of Animal Husbandry and Veterinary, \\ Hubei Academy of Agricultural Science, China \\ 2 RNA Group, College of Life Science, Wuhan University, China \\ 3 The Third Affiliated Hospital of Sun Yat-Sen University, Tianhe District, China
}

Corresponding author: biyanzhen@gmail.com

Received October 27, 2010 / Accepted January 19, 2011

Published online: March 15, 2011

(C) 2011 by Pontificia Universidad Católica de Valparaíso, Chile

\begin{abstract}
Background: As key gene regulators, microRNAs post-transcriptionally modulate gene expression via binding to partially complementary sequence in the 3' UTR of target mRNA. An accurate, rapid and quantitative tool for sensing and validation of miRNA targets is of crucial significance to decipher the functional implications of miRNAs in cellular pathways.

Results: Taking advantage of an improved restriction-free cloning method, we engineered a novel built-in dual luciferase reporter plasmid where Firefly and Renilla luciferase genes were assembled in a single plasmid named "pFila". This design eliminates the transfection of a separate control plasmid and thus minimizes the time and labor required for miRNA-target sensing assays. pFila consistently produces Firefly and Renilla luciferase activities when transfected into human-, monkey- and mousederived mammalian cell systems. Moreover, pFila is capable of recapitulating the interaction of miR-16 and its known target CCNE1 in Hela cells. Additionally, pFila is shown to be a sensitive miR-biosensor by evaluating the inhibition efficiency of endogenous miRNA.

Conclusions: pFila would facilitate miRNA target identification and verification in a rapid and simplified manner. Also, pFila is a sensitive biosensor for active miRNA profiling in vivo.
\end{abstract}

Keywords: biosensor, luciferase, ligase-independent, miRNA, target

\section{INTRODUCTION}

MicroRNAs are key post-transcriptional regulators of gene expression in a variety of cellular events. They mediate translational repression, and sometimes destabilization, of target mRNAs by directing miRISC (microRNA-induced silencing complex) to imperfect complementary sequences in 3' UTR (Bartel, 2009). It has been predicted that more than $60 \%$ of human genes are putative targets of one or more miRNAs, while it is also suggested that an individual miRNA is capable of regulating multiple target mRNAs (Backes et al. 2010). Consequently, a major challenge in miRNA study is the experimental identification and validation of its functional target(s). Various reporter systems have been developed to probe the interaction between individual miRNA and its target (Lee et al. 2008), of which dual luciferase assay is widely adopted to achieve this end (Brennecke et al. 2005). The current dual luciferase assay encompasses two separate plasmids, one containing the region of interest and the other serving as internal control to normalize transfection variation (Robertson et al. 2010). A major drawback of this system is the tedious steps for preparing and transfecting control vector. A more convenient reporter is desired to simplify the conventional protocol. 
Bi et al.

In addition, there are several methods, including northern blotting, real-time PCR, microarray and deep sequencing, to quantify miRNAs (Willenbrock et al. 2009). However, these techniques simply output the homeostasis of endogenous miRNAs other than active molecules. Moreover, these approaches are either time-consuming and laborious or cost-ineffective. Therefore, a convenient and quantitative miRNA biosensor is desired to measure functional miRNA in vivo.

In this study, by taking advantage of ligase-free homologous recombination in $E$. coli, we engineered a novel reporter that integrated Firefly and Renilla luciferase genes (Fluc and Rluc, same below) in a single plasmid. Its expressivity and applicability were further examined to demonstrate that this novel reporter will facilitate the screening and sensing of miRNAs and their targets in a simplified and precise manner.

\section{MATERIALS AND METHODS}

\section{DNA and RNA oligos}

Unless stated elsewhere, all DNA and RNA oligos are presented as 5' $\rightarrow 3$ ' direction. Primers for amplifying Fluc gene were Pf, AAGGATCCAGGTGGCACTTTTCG TGCGATCTGCATCTCAATTAG; $\mathrm{Pr}$, GAAAAATAAACAAATAGGGGTTCCGCGCAC CTCACATGTTCTTTCCTGC (sequence annealing to Fluc gene was shown in boldface; sequence complementary to insertion site on pRL-TK was underlined). 3' outermost primer P2R was CGAAAAGTGCCACCTGGATCCTT. Sequencing primers for pFila was SF, GATGCACCTG ATGAAATGGG; SR, AGGACAGGTG CCGGCAGCGC. For creation of Apal site, see details in reference (Wang et al. 2009). RNA oligos were chemically synthesized and purified by Genepharma Co. Ltd., (Shanghai, P.R. China). Human miR16-1 was sense UAGCAGCACGUAAAUAUUGGCG and antisense CGCCAAUAUUUACGUGCUGCUA. Negative control for miRNA mimics was sense UUGUACUACACAAAAGUACUG and antisense CAGUACUUUUGUGUAGUACAA. SIRNA against Rluc mRNA was sense GUAGCGCGGUGUAUUAUACdTdT and antisense GUAUAAUACACCGCGCUACdTdT. Methylated anti-miR-16-1 inhibitor CGC CAA UAU UUACGU GCU GCU A, scramble anti-miR control UUG UAC UAC ACA AAA GUA CUG.

\section{Construction of plasmids}

pFila was fabricated by bridging-PCR coupled with homologous recombination in bacteria. A duplex bridging PCR was conducted in a $50 \mu \mathrm{l}$ mixture: Pf $4 \mu \mathrm{l}(250 \mathrm{nM}), \operatorname{Pr} 2 \mu \mathrm{l}(125 \mathrm{nM}), \mathrm{P} 2 \mathrm{R}$ primer $2 \mu \mathrm{l}$ (125 nM), pGL3-promoter plasmid $1 \mu \mathrm{l}(5 \mathrm{ng})$, modified pRL-TK plasmid $1 \mu \mathrm{l}$ (10 ng or $50 \mathrm{ng}$ ), $2 \mathrm{mM}$ dNTP $5 \mu \mathrm{l}, 25 \mathrm{mM} \mathrm{MgSO} 42 \mu \mathrm{l}, 10$ x KOD buffer $5 \mu \mathrm{l}$, KOD plus $1 \mu \mathrm{l}(1$ unit), PCR-grade water $27 \mu \mathrm{l}$. The condition was: $95^{\circ} \mathrm{C} 2 \mathrm{~min}, 30$ cycles of $\left(95^{\circ} \mathrm{C} 15 \mathrm{sec}, 55^{\circ} \mathrm{C} 30 \mathrm{sec}, 68^{\circ} \mathrm{C} 6.5 \mathrm{~min}\right)$. The PCR products were digested with $\mathrm{Dphl}$ (Fermentas, Lithuania) at $37^{\circ} \mathrm{C}$ for $2 \mathrm{hrs}$ to destroy methylated plasmids while keeping the nascent DNA intact with the following reaction: PCR products, $26 \mu$ l; $10 \mathrm{x}$ Tango buffer $3 \mu \mathrm{l}$; Dpn I $1 \mu \mathrm{l}$ (1 unit). An aliquot of $5 \mu$ ldigested PCR products were transformed into $E$. coli $\mathrm{DH} 5 \alpha$ to generate recombinants that were subsequently sequenced to verify the integrity. Pf and Pr primer pair was used to amplify Fluc gene composed of SV40 promoter, Fluc coding region and SV40 late poly(A) signal. Fluc gene was designed to fuse into a modified pRL-TK plasmid downstream of 3'UTR of RluC and upstream of beta-lactamase gene. For the sequence context of human CCNE1 3'UTR, see details in reference (Wang et al. 2009). The wild-type and mutated human CCNE1 target regions were sub-cloned into pFila with $X b a I$ and $A$ pa $I$.

\section{Cell culture, transfection and dual luciferase assay}

Human cervical carcinoma Hela cells, African green monkey kidney Vero cells and mouse myoblast $\mathrm{C} 2 \mathrm{C} 12$ cells were maintained in high glucose DMEM (Invitrogen) supplemented with $10 \%$ fetal calf serum (Gibco) at $37^{\circ} \mathrm{C}$ and $5 \% \mathrm{CO}_{2} .4 \times 10^{4}$ cells were seeded in a 24 -well plate one day before transfection. For miRNA mimics and plasmid co-transfection, $1 \mu \mathrm{l} 20 \mu \mathrm{M}$ chemically synthesized miR16 mimics and $50 \mathrm{ng}$ pFila (pFila-CCNE1-wildtype and pFila-CCNE1-mut1\&2) or $50 \mathrm{ng}$ pGL3-promoter (internal control) and $\mathrm{pRL}-\mathrm{ML}$ plasmids (pRL-ML-CCNE1-wildtype and pRL-ML-CCNE1-mut1\&2; $25 \mathrm{ng}$ each) were mixed with $2 \mu \mathrm{l}$ Lipofectamine2000 (Invitrogen) as transfection complex. For evaluation of endogenous miRNA inhibition with pFila, 20 nM 2'-O-methylated anti-human miR16-1 inhibitor was transfected into Hela cells by $0.5 \mu$ l Lipofectamine2000 (Invitrogen), RNAiMAX (Invitrogen), Sofast 
(Sunmabio, China), Fugene (Roche), respectively. All transfections were performed in three independent experiments with each in triplicate. A DLR ${ }^{\mathrm{TM}}$ Assay (Promega) was adopted to measure luciferase activity in a Glomax luminometer essentially according to manufacturer's instruction.

\section{Statistical analysis}

Luciferase levels were reported as ratio over that observed in control transfections, where R/uc activities were normalized to Fluc activities. The data represented the mean \pm S.D. of three independent experiments and were analyzed by Student's $t$-test. Differences below $p<0.01$ were regarded as significant.

\section{Accession number}

The sequence and annotation of pFila has been deposited in Genbank with accession number HQ425563. pFila is freely available upon request.

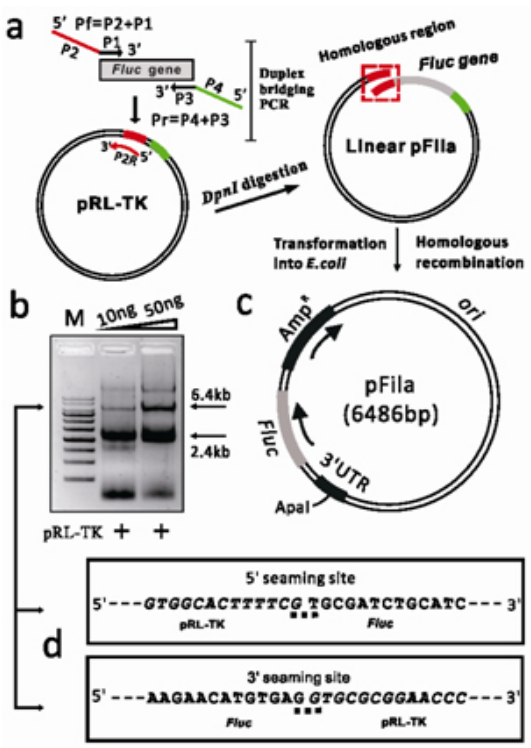

Fig. 1 Concept and engineering of pFila. (a) In this reaction, the primary process was the production of Fluc gene by $\mathrm{Pf}(\mathrm{Pf}$ is composed of $\mathrm{P} 1$ and $\mathrm{P} 2$, which are complementary to upstream of Fluc gene and pRL-TK plasmid, respectively) and $\operatorname{Pr}(\mathrm{Pr}$ is composed of $\mathrm{P} 3$ and $\mathrm{P} 4$, which are complementary to downstream of Fluc gene and pRLTK plasmid, respectively) primers. In the secondary process, amplified Fluc gene bearing a deliberately designed region annealed with its homologous sequence in $\mathrm{pRL}-\mathrm{TK}$, thus generating a large linear fragment. In order to exponentially amplify the linear fragment, the outermost Pf and P2R (complementary to P2) primer pair initiated the ternary process to produce adequate fused fragment. (b) Duplex bridge PCR. The $2.4 \mathrm{~kb}$ fragment was Fluc gene, while the $6.4 \mathrm{~kb}$ fragment was the desired fusion product. M is $1 \mathrm{~kb}$ DNA ladder. (c) Map of pFila. The engineered pFila is $6486 \mathrm{bp}$ in length. Xbal and Apal restriction sites are located in the 3'UTR of R/uc to facilitate the cloning of miRNA target for reporter assay. (d) 5' and 3' seaming sites of Fluc into pRL-TK. For detailed information, please refer to Gene sequence 1.

\section{RESULTS AND DISCUSSION}

The complicated procedures of the current dual luciferase reporter assay for miRNA target screening prompted us to upgrade its practicality for simplified manipulations. Specifically, we aimed to integrate Fluc and Rluc genes in a single vector. As restriction sites were not available to sub-clone Fluc gene to pRL-TK plasmid with ligase-dependent method, we adopted an improved restriction-free gene fusion approach inspired by the principle of site-directed mutagenesis (Zheng et al. 2004). As shown in Figure $1 \mathrm{a}$ and Figure 1b, a duplex bridging PCR was carried out to produce the $6.4 \mathrm{~kb}$ linear fusion fragment 
with homologous sequences at both 5' and 3' ends. The PCR products were digested by Dpnl and transformed into $E$. coli to achieve the circular plasmid based on homologous recombination. Sequencing of the recombinant (named pFila) revealed that Fluc gene had been successfully fused into pRL-TK plasmid at designed location (Figure 1c and Figure 1d; Gene sequence 1).

We then evaluated the expressivity of pFila in different mammalian systems. As presented in Figure $2 a$, luminescence of pFila was reported in a wide linear range when transfected into human-, mouseand monkey-sourced cell lines at gradient amounts, indicating that pFila consistently produces luciferases in vivo. This also implies that the ordered assembly of Fluc and Rluc luciferase genes in pRL-TK plasmid does not interfere with their individual expression. Next, the applicability and reproducibility of pFila were examined by recapitulating the regulation of human miR16-1 and its known target CCNE1 (Wang et al. 2009). miR16-1 mimics down-regulated the Rluc activity fused with wildtype CCNE1 3'UTR but not a mutant 3'UTR (Figure 2b); the latter carried altered residues that were introduced in the miR16-1 "seed-pairing" recognition site (Figure 2b). This observation perfectly photocopied the result that was achieved by traditional dual reporter assay (Figure $2 \mathrm{~b}$ ), indicating that pFila as a more convenient reporter is fully applicable to miRNA functional analysis. Finally, we applied pFila carrying the wild-type and mutant 3'TUR of CCNE1 to assessing the blockage efficiency of endogenous miR16-1. 2'-O-methylated anti-miRNA-16-1 RNA oligo was transfected by four types of transfection reagents, i.e. Lipofectamine2000, RNAiMAX, Fugene, Sofast. Inhibition efficacy of endogenous miR16-1 varied, of which RNAiMAX achieved the most potent blocking effect. This assay implies that pFila is a sensitive miRNA biosensor to reflect the level of functional miRNAs. It also suggests that the choice of delivery method is an important determinant when conducting loss-offunction analysis of miRNAs.

In summary, we have successfully engineered a novel dual luciferase plasmid that incorporated Fluc and Rluc genes in a single vector, allowing the simultaneous expression of both luciferase genes. This improvement maintains comparable reproducibility but minimizes the time and labor required in conventional dual luciferase protocol. Furthermore, several lines of evidence were presented to demonstrate its application in miRNA functional analysis. These results indicate that pFila will find its wide application in the screening, identification and validation of miRNA with its potential mRNA targets.

a

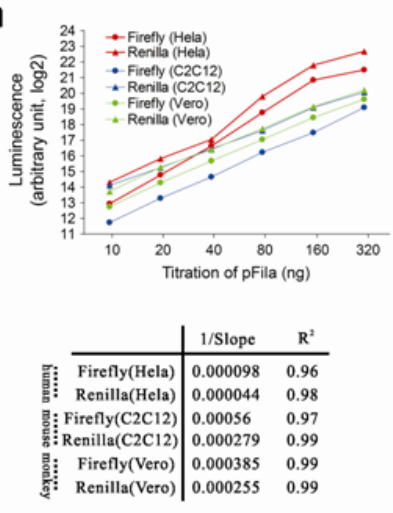

b

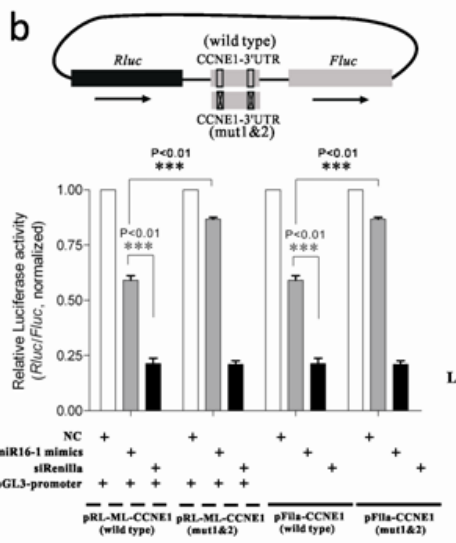

C

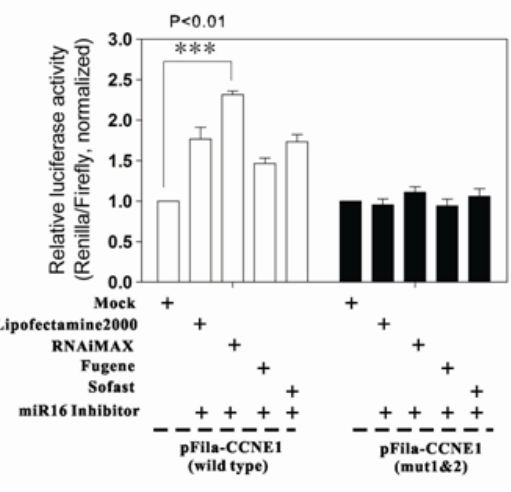

Fig. 2 Expressivity and application of pFila. (a) pFila consistently produces Fluc and R/uc activities in human-, mouse- and monkey-sourced mammalian cell lines. pFila was gradientlytransfected into the cells stated above and Fluc and Rluc luciferase levels were determined by a DLR ${ }^{\mathrm{TM}}$ Assay (Promega). As shown in this graph, Fluc and Rluc activities were in a linear range for all the selected points. (b) pFila is capable of recapitulating the interaction of miR16 and its known target CCNE1. Wild-type and mutant CCNE1 3'UTRs were sub-cloned into pFila and cotransfected with miR16-1 mimics. Conventional dual luciferase reporter was conducted in parallel to compare their reproducibility. siRNA against Rluc serves as positive control. Rluc with mutant CCNE1 3'UTR was rescued in comparison with pFila-CCNE1-3'UTR-wildtype, indicating that CCNE1 is a direct target of miR16 as previously reported (Wang et al. 2009). (c) miR16-1 inhibitors were co-transfected with pFila-CCNE1-3'UTR-wildtype or mutant plasmids into Hela cells by different transfection reagents to block endogenous miR16-1. Inhibition efficacy varies with the selected agents, where RNAiMAX achieved the most potent inhibition. It implies that pFila is a sensitive biosensor for functional miRNA profiling. 
Gene sequence 1. Sequencing of the seaming sites: sequence annealing to Fluc gene was shown in boldface; sequence complementary to insertion site on PRL-TK was underlined.

\author{
Seq.1 (sequencing primer SF: GATGCACCTGATGAAATGGG )
}

TTTTTAAATCCAATTCGGTTGAACGAGTTCTCAAAAATGAACAATAATTCTAGATTCCGAGATATCGGTAATGGGC CCTAGAGCGGCCGCTTCGAGCAGACATGATAAGATACATTGATGAGTTTGGACAAACCACAACTAGAATGCAGTGA AAAAAATGCTTTATTTGTGAAATTTGTGATGCTATTGCTTTATTTGTAACCATTATAAGCTGCAATAAACAAGTTA ACAACAACAATTGCATTCATTTTATGTTTCAGGTTCAGGGGGAGGTGTGGGAGGTTTTTTAAAGCAAGTAAAACCT CTACAAATGTGGTAAAATCGATAAGGATCCAGGTGGCACTTTTCGTGCGATCTGCATCTCAATTAGTCAGCAACCA TAGTCCCGCCCCTAACTCCGCCCATCCCGCCCCTAACTCCGCCCA GTTCCGCCCATTCTCCGCCCCATCGCTGACT AATTTTTTTTATTTATGCAGAGGCCGAGGCCGCCTCGGCCTCTGAGCTATTCCAGAAGTAGTGAGGAGGCTTTTTT GGAGGCCTAGGCTTTTGCAAAAAGCTTGGCATTCCGGTACTGTTGGTAAAGCCACCATGGAAGACGCCAAAAACAT AAAGAAAGGCCCGGCGCCATTCTATCCGCTGGAAGATGGAACCGCTGGAGAGCAACTGCATAAGGCTATGAAGAGA TACGCCCTGGTTCCTGGAACAATTGCTTTTACAGATGCACATATCGAGGTGGACATCACTTACGCTGAGTACTTCG AAATGTCCGTTCGGTTGGCAGAAGCTATGAAACGATATGGGCTGAATACAAATCACAGAATCGTCGTATGCAGTGA AAACTCTCTTCAATTCTTTATGCCGGTGTTGGGCGCGTTATTTATCGGAGTTGCAGTTGCGCCCGCGAACGACATT TATAATGAACGTGAATTGCTCAACAGTATGGGCATTTCGCAGCCTACCGTGGTGTTCGTTTCCAAAAAGGGTTGCA AAAAATTTTGAACGTGCAAAAAAGCTCCCAATCATCCAAAAAATTATTATCATGGATTCTAAAACGGATTACCAGG GGATTCAGTCGATGTACACGTCGTCACATCTCATCTACCTTCCCGGTTTATGATACGATTTTGTGCAAGAGTCTTC GATAGGACAAGACAATTGCACTGATCATGACCTCTCTGATCTACTGGACTGCTAGGTGCGACCTTGTCTCATAGAC TGCCTGGCCGGTAGAAATTCTCGCC

\title{
Seq.2 (sequencing primer SR: AGGACAGGTG CCGGCAGCGC)
}

CCTGGAAATAAGAGGTGAGGTGATCATCAGGTAATGGGGTACTGTCGACGATACCCAATGCTATCAGGAGGCACGT TATTCAGCGATCTGTCTATTCGGTCATCATAGTTGCTGACTCCCGTCGGTAGATAACTACGATACGGAGGCTACAA TCTGCCCAGTGCTGCATGATACGCGAGACCACGCTCACCGGCTCAGATTATCAGCATAACAGCAGCGAGGGCCGAG CGCAGAGTGTCTGCACTTATCCGCTCCATCCAGTCTATAATGTGCGGGAAGCTAGAGTAAGTAGTTCGCCAGTTAA TAGTTTGCGCAACGTTGTTGCCATTGCTACAGGCATCGTGGTGTCACGCTCGTCGTTTGGTATGGCTTCATTCAGC TCCGGTTCCCAACGATCAAGGCGAGTTACATGATCCCCCATGTTGTGCAAAAAAGCGGTTAGCTCCTTCGGTCCTC CGATCGTTGTCAGAAGTAAGTTGGCCGCAGTGTTATCACTCATGGTTATGGCAGCACTGCATAATTCTCTTACTGT* / CATGCCATCCGTAAGATGCTTTTCTGTGACTGGTGAGTACTCAACCAAGTCATTCTGAGAATAGTGTATGCGGCGA CCGAGTTGCTCTTGCCCGGCGTCAATACGGGATAATACCGCGCCACATAGCAGAACTTTAAAAGTGCTCATCATTG GAAAACGTTCTTCGGGGCGAAAACTCTCAAGGATCTTACCGCTGTTGAGATCCAGTTCGATGTAACCCACTCGTGC ACCCAACTGATCTTCAGCATCTTTTACTTTCACCAGCGTTTCTGGGTGAGCAAAAACAGGAAGGCAAAATGCCGCA AAAAAGGGAATAAGGGCGACACGGAAATGTTGAATACTCATACTCTTCCTTTTTCAATATTATTGAAGCATTTATC AGGGTTATTGTCTCATGAGCGGATACATATTTGAATGTATTTAGAAAAATAAACAAATAGGGGTTCCGCGCACCTC ACATGTTCTTTCCTGCGTTATCCCCTGATTCTGTGGATAACCGTATTACCGCCTTTGAGTGAGCTGATACCGCTCG CCGCAGCCGAACGACCGAGCGCAGCGAGTCAGAGCGAGTGGGG

Seq.3 pFila sequence

\begin{tabular}{lcc} 
Base pair: & $6486 \mathrm{bp}$ \\
HSV TK promoter & $7-759$ & \\
Chimeric intron & $826-962$ & \\
T7 RNA polymerase Promoter (-17 to +2) & $1006-1024$ \\
T7 RNA polymerase transcription & 1023 \\
Rluc reporter gene & $1034-1969$ & \\
Xbal restriction site & $1971-1976$ \\
Apal restriction site & $1995-2000$ \\
SV40 late polyadenylation signal(upstream) & $2039-2240$ \\
SV40 promoter & $2279-2481$ \\
Fluc reporter gene & $2511-4163$ \\
SV40 late polyadenylation signal(downstream) & $4195-4416$ \\
Beta-lactamase (AmpR) & $4800-5660$ \\
pBR322 plasmid replication origin & \multicolumn{2}{c}{$5802-6445$}
\end{tabular}

1 AgATCTAAAT GAGTCTTCGg ACCTCGCGGg GGCCGCTTAA GCGGTGGTTA

51 GGGTTTGTCT GACGCGGGGG GAgGgGGAAG GAACGAAACA CTCTCATTCG

101 GAGGCGGCTC GGGGTTTGGT CTTGGTGGCC ACGGGCACGC AGAAGAGCGC

151 CGCGATCCTC TTAAGCACCC CCCCGCCCTC CGTGGAGGCG GGGGTTTGGT

201 CGGCGGGTGG TAACTGGCGG GCCGCTGACT CGGGCGGGTC GCGCGCCCCA

251 GAGTGTGACC TTTTCGGTCT GCTCGCAGAC CCCCGGGCGG CGCCGCCGCG

301 GCGGCGACGG GCTCGCTGGG TCCTAGGCTC CATGGGGACC GTATACGTGG

351 ACAGGCTCTG GAGCATCCGC ACGACTGCGG TGATATTACC GGAGACCTTC

401 TGCGGGACGA GCCGGGTCAC GCGGCTGACG CGGAGCGTCC GTTGGGCGAC 
Bi et al.

451 AAACACCAGG ACGGGGCACA GGTACACTAT CTTGTCACCC GGAGGCGCGA 501 GGGACTGCAG GAGCTTCAGG GAGTGGCGCA GCTGCTTCAT CCCCGTGGCC 551 CGTTGCTCGC GTTTGCTGGC GGTGTCCCCG GAAGAAATAT ATTTGCATGT 601 CTTTAGTTCT ATGATGACAC AAACCCCGCC CAGCGTCTTG TCATTGGCGA 651 ATTCGAACAC GCAGATGCAG TCGGGGCGGC GCGGTCCCAG GTCCACTTCG 701 CATATTAAGG TGACGCGTGT GGCCTCGAAC ACCGAGCGAC CCTGCAGCGA 751 CCCGCTTAAA AGCTTGATTC TTCTGACACA ACAGTCTCGA ACTTAAGCTG 801 CAGAAGTTGG TCGTGAGGCA CTGGGCAGGT AAGTATCAAG GTTACAAGAC 851 AGGTTTAAGG AGACCAATAG AAACTGGGCT TGTCGAGACA GAGAAGACTC 901 TTGCGTTTCT GATAGGCACC TATTGGTCTT ACTGACATCC ACTTTGCCTT 951 TCTCTCCACA GGTGTCCACT CCCAGTTCAA TTACAGCTCT TAAGGCTAGA 1001 GTACTTAATA CGACTCACTA TAGGCTAGCC ACCATGACTT CGAAAGTTTA 1051 TGATCCAGAA CAAAGGAAAC GGATGATAAC TGGTCCGCAG TGGTGGGCCA 1101 GATGTAAACA AATGAATGTT CTTGATTCAT TTATTAATTA TTATGATTCA 1151 GAAAAACATG CAGAAAATGC TGTTATTTTT TTACATGGTA ACGCGGCCTC 1201 TTCTTATTTA TGGCGACATG TTGTGCCACA TATTGAGCCA GTAGCGCGGT 1251 GTATTATACC AGACCTTATT GGTATGGGCA AATCAGGCAA ATCTGGTAAT 1301 GGTTCTTATA GGTTACTTGA TCATTACAAA TATCTTACTG CATGGTTTGA 1351 ACTTCTTAAT TTACCAAAGA AGATCATTTT TGTCGGCCAT GATTGGGGTG 1401 CTTGTTTGGC ATTTCATTAT AGCTATGAGC ATCAAGATAA GATCAAAGCA 1451 ATAGTTCACG CTGAAAGTGT AGTAGATGTG ATTGAATCAT GGGATGAATG 1501 GCCTGATATT GAAGAAGATA TTGCGTTGAT CAAATCTGAA GAAGGAGAAA 1551 AAATGGTTTT GGAGAATAAC TTCTTCGTGG AAACCATGTT GCCATCAAAA 1601 ATCATGAGAA AGTTAGAACC AGAAGAATTT GCAGCATATC TTGAACCATT 1651 CAAAGAGAAA GGTGAAGTTC GTCGTCCAAC ATTATCATGG CCTCGTGAAA 1701 TCCCGTTAGT AAAAGGTGGT AAACCTGACG TTGTACAAAT TGTTAGGAAT 1751 TATAATGCTT ATCTACGTGC AAGTGATGAT TTACCAAAAA TGTTTATTGA 1801 ATCGGACCCA GGATTCTTTT CCAATGCTAT TGTTGAAGGT GCCAAGAAGT 1851 TTCCTAATAC TGAATTTGTC AAAGTAAAAG GTCTTCATTT TTCGCAAGAA 1901 GATGCACCTG ATGAAATGGG AAAATATATC AAATCGTTCG TTGAGCGAGT 1951 TCTCAAAAAT GAACAATAAT TCTAGATTCC GAGATATCGG TAATGGGCCC 2001 TAGAGCGGCC GCTTCGAGCA GACATGATAA GATACATTGA TGAGTTTGGA 2051 CAAACCACAA CTAGAATGCA GTGAAAAAAA TGCTTTATTT GTGAAATTTG 2101 TGATGCTATT GCTTTATTTG TAACCATTAT AAgCTGCAAT AAACAAGTTA 2151 ACAACAACAA TTGCATTCAT TTTATGTTTC AGGTTCAGGG GGAGGTGTGG 2201 GAGGTTTTTT AAAGCAAGTA AAACCTCTAC AAATGTGGTA AAATCGATAA 2251 GGATCCAGGT GGCACTTTTC GTGCGATCTG CATCTCAATT AGTCAGCAAC 2301 CATAGTCCCG CCCCTAACTC CGCCCATCCC GCCCCTAACT CCGCCCAGTT 2351 CCGCCCATTC TCCGCCCCAT CGCTGACTAA TTTTTTTTAT TTATGCAGAG 2401 GCCGAGGCCG CCTCGGCCTC TGAGCTATTC CAGAAGTAGT GAGGAGGCTT 2451 TTTTGGAGGC CTAGGCTTTT GCAAAAAGCT TGGCATTCCG GTACTGTTGG 2501 TAAAGCCACC ATGGAAGACG CCAAAAACAT AAAGAAAGGC CCGGCGCCAT 2551 TCTATCCGCT GGAAGATGGA ACCGCTGGAg AgCAACTGCA TAAGGCTATG 2601 AAGAGATACG CCCTGGTTCC TGGAACAATT GCTTTTACAG ATGCACATAT 2651 CGAGGTGGAC ATCACTTACG CTGAGTACTT CGAAATGTCC GTTCGGTTGG 2701 CAGAAGCTAT GAAACGATAT GGGCTGAATA CAAATCACAG AATCGTCGTA 2751 TGCAGTGAAA ACTCTCTTCA ATTCTTTATG CCGGTGTTGG GCGCGTTATT 2801 TATCGGAGTT GCAGTTGCGC CCGCGAACGA CATTTATAAT GAACGTGAAT 2851 TGCTCAACAG TATGGGCATT TCGCAGCCTA CCGTGGTGTT CGTTTCCAAA 2901 AAGGGGTTGC AAAAAATTTT GAACGTGCAA AAAAAGCTCC CAATCATCCA 2951 AAAAATTATT ATCATGGATT CTAAAACGGA TTACCAGGGA TTTCAGTCGA 3001 TGTACACGTT CGTCACATCT CATCTACCTC CCGGTTTTAA TGAATACGAT 3051 TTTGTGCCAG AGTCCTTCGA TAGGGACAAG ACAATTGCAC TGATCATGAA 3101 CTCCTCTGGA TCTACTGGTC TGCCTAAAGg TGTCGCTCTG CCTCATAGAA 3151 CTGCCTGCGT GAGATTCTCG CATGCCAGAG ATCCTATTTT TGGCAATCAA 3201 ATCATTCCGG ATACTGCGAT TTTAAGTGTT GTTCCATTCC ATCACGGTTT 3251 TGGAATGTTT ACTACACTCG GATATTTGAT ATGTGGATTT CGAGTCGTCT 3301 TAATGTATAG ATTTGAAGAA GAGCTGTTtC TGAGGAGCCT TCAGGATTAC 3351 AAGATTCAAA GTGCGCTGCT GGTGCCAACC CTATTCTCCT TCTTCGCCAA 3401 AAGCACTCTG ATTGACAAAT ACGATTTATC TAATTTACAC GAAATTGCTT 3451 CTGGTGGCGC TCCCCTCTCT AAGGAAGTCG GGGAAGCGGT TGCCAAGAGG 3501 TTCCATCTGC CAGGTATCAG GCAAGGATAT GGGCTCACTG AGACTACATC 3551 AGCTATTCTG ATTACACCCG AGGGGGATGA TAAACCGGGC GCGGTCGGTA 3601 AAGTTGTTCC ATTTTTTGAA GCGAAGGTTG TGGATCTGGA TACCGGGAAA 3651 ACGCTGGGCG TTAATCAAAG AGGCGAACTG TGTGTGAGA GTCCTATGAT 3701 TATGTCCGGT TATGTAAACA ATCCGGAAGC GACCAACGCC TTGATTGACA 3751 AgGATGGATG GCTACATTCT GGAGACATAG CTTACTGGGA CGAAGACGAA 3801 CACTTCTTCA TCGTTGACCG CCTGAAGTCT CTGATTAAGT ACAAAGGCTA 3851 TCAGGTGGCT CCCGCTGAAT TGGAATCCAT CTTGCTCCAA CACCCCAACA 3901 TCTTCGACGC AGGTGTCGCA GGTCTTCCCG ACGATGACGC CGGTGAACTT 3951 CCCGCCGCCG TTGTTGTTTT GGAGCACGGA AAGACGATGA CGGAAAAAGA 
4001 GATCGTGGAT TACGTCGCCA GTCAAGTAAC AACCGCGAAA AAgTTGCGCG 4051 GAGGAGTTGT GTTTGTGGAC GAAGTACCGA AAGGTCTTAC CGGAAAACTC 4101 GACGCAAGAA AAATCAGAGA GATCCTCATA AAGGCCAAGA AGGGCGGAAA 4151 GATCGCCGTG TAATTCTTGA GTCGGGGCGG CCGGCCGCTT CGAGCAGACA 4201 TGATAAGATA CATTGATGA TTTGGACAAA CCACAACTAG AATGCAGTGA 4251 AAAAAATGCT TTATTTGTGA AATTTGTGAT GCTATTGCTT TATTTGTAAC 4301 CATTATAAGC TGCAATAAAC AAGTTAACAA CAACAATTGC ATTCATTTTA 4351 TGTTTCAGGT TCAGGGGGAG GTGTGGGAGg TTTTTTAAAG CAAGTAAAAC 4401 CTCTACAAAT GTGGTAAAAT CGATAAGGAT CCGTCGACCG ATGCCCTTGA 4451 GAGCCTTCAA CCCAGTCAGC TCCTTCCGGT GGGCGCGGGG CATGACTATC 4501 GTCGCCGCAC TTATGACTGT CTTCTTTATC ATGCAACTCG TAGGACAGGT 4551 GCCGGCAGCG CTCTTCCGCT TCCTCGCTCA CTGACTCGCT GCGCTCGGTC 4601 GTTCGGCTGC GGCGAGCGGT ATCAGCTCAC TCAAAGGCGG TAATACGGTT 4651 ATCCACAGAA TCAGGGGATA ACGCAGGAAA GAACATGTGA GGTGCGCGGA 4701 ACCCCTATTT GTTTATTTTT CTAAATACAT TCAAATATGT ATCCGCTCAT 4751 GAGACAATAA CCCTGATAAA TGCTTCAATA ATATTGAAAA AGGAAGAGTA 4801 TGAGTATTCA ACATTTCCGT GTCGCCCTTA TTCCCTTTTT TGCGGCATTT 4851 TGCCTTCCTG TTTTTGCTCA CCCAGAAACG CTGGTGAAAG TAAAAGATGC 4901 TGAAGATCAG TTGGGTGCAC GAGTGGGTTA CATCGAACTG GATCTCAACA 4951 GCGGTAAGAT CCTTGAGAGT TTTCGCCCCG AAGAACGTTT TCCAATGATG 5001 AGCACTTTTA AAGTTCTGCT ATGTGGCGCG GTATTATCCC GTATTGACGC 5051 CGGGCAAGA CAACTCGGTC GCCGCATACA CTATTCTCAG AATGACTTGG 5101 TTGAGTACTC ACCAGTCACA GAAAAGCATC TTACGGATGG CATGACAGTA 5151 AGAGAATTAT GCAGTGCTGC CATAACCATG AGTGATAACA CTGCGGCCAA 5201 CTTACTTCTG ACAACGATCG GAGGACCGAA GGAGCTAACC GCTTTTTTGC 5251 ACAACATGGG GGATCATGTA ACTCGCCTTG ATCGTTGGGA ACCGGAGCTG 5301 AATGAAGCCA TACCAAACGA CGAGCGTGAC ACCACGATGC CTGTAGCAAT 5351 GGCAACAACG TTGCGCAAAC TATTAACTGG CGAACTACTT ACTCTAGCTT 5401 CCCGGCAACA ATTAATAGAC TGGATGGAGg CGGATAAAGT TGCAGGACCA 5451 CTTCTGCGCT CGGCCCTTCC GGCTGGCTGG TTTATTGCTG ATAAATCTGG 5501 AGCCGGTGAG CGTGGGTCTC GCGGTATCAT TGCAGCACTG GGGCCAGATG 5551 GTAAGCCCTC CCGTATCGTA GTTATCTACA CGACGGGGAG TCAGGCAACT 5601 ATGGATGAAC GAAATAGACA GATCGCTGAG ATAGGTGCCT CACTGATTAA 5651 GCATTGGTAA CTGTCAGACC AAGTTTACTC ATATATACTT TAGATTGATT 5701 TAAAACTTCA TTTTTAATTT AAAAGGATCT AGGTGAAGAT CCTTTTTGAT 5751 AATCTCATGA CCAAAATCCC TTAACGTGAG TTTTCGTTCC ACTGAGCGTC 5801 AGACCCCGTA GAAAAGATCA AAGGATCTTC TTGAGATCCT TTTTTTCTGC 5851 GCGTAATCTG CTGCTTGCAA ACAAAAAAAC CACCGCTACC AGCGGTGGTT 5901 TGTTTGCCGG ATCAAGAGCT ACCAACTCTT TTTCCGAAGG TAACTGGCTT 5951 CAGCAGAGCG CAGATACCAA ATACTGTTCT TCTAGTGTAG CCGTAGTTAG 6001 GCCACCACTT CAAGAACTCT GTAGCACCGC CTACATACCT CGCTCTGCTA 6051 ATCCTGTTAC CAGTGGCTGC TGCCAGTGGC GATAAGTCGT GTCTTACCGG 6101 GTTGGACTCA AGACGATAGT TACCGGATAA GGCGCAGCGG TCGGGCTGAA 6151 CGGGGGGTTC GTGCACACAG CCCAGCTTGG AGCGAACGAC CTACACCGAA 6201 CTGAGATACC TACAGCGTGA GCTATGAGAA AGCGCCACGC TTCCCGAAGG 6251 GAGAAAGGCG GACAGGTATC CGGTAAGCGG CAGGGTCGGA ACAGGAGAGC 6301 GCACGAGGGA GCTTCCAGGG GGAAACGCCT GGTATCTTTA TAGTCCTGTC 6351 GGGTTTCGCC ACCTCTGACT TGAGCGTCGA TTTTTGTGAT GCTCGTCAGG 6401 GGGGCGGAGC CTATGGAAAA ACGCCAGCAA CGCGGCCTTT TTACGGTTCC 6451 TGGCCTTTTG CTGGCCTTTT GCTCACATGG CTCGAC

\section{ACKNOWLEDGMENTS}

We appreciated the technical assistance from Cell Signaling Lab of Wuhan University.

Financial support: This work was funded by a research grant from Hubei Key Laboratory of Animal Embryo Engineering and Molecular Breeding to Y. Z. Bi (2010ZD163).

\section{REFERENCES}

BACKES, C.; MEESE, E.; LENHOF, H.P. and KELLER, A. (2010). A dictionary on microRNAs and their putative target pathways. Nucleic Acids Research, vol. 38, no. 13, p. 4476-4486. [CrossRef]

BARTEL, D.P. (2009). MicroRNAs: Target recognition and regulatory functions. Cell, vol. 136, no. 2, p. 215-233. [CrossRef] 
BRENNECKE, J.; STARK, A.; RUSSELL, R.B. and COHEN, S.M. (2005). Principles of microRNA-target recognition. PLoS Biology, vol. 3, no. 3, p. e85. [CrossRef]

LEE, J.Y.; KIM, S.; HWANG, D.W.; JEONG, J.M.; CHUNG, J.K.; LEE, M.C. and LEE, D.S. (2008). Development of a dual-luciferase reporter system for in vivo visualization of microRNA biogenesis and posttranscriptional regulation. The Journal of Nuclear Medicine, vol. 49, no. 2, p. 285-294. [CrossRef]

ROBERTSON, B.; DALBY, A.B.; KARPILOW, J.; KHVOROVA, A.; LEAKE, D. and VERMEULEN, A. (2010). Specificity and functionality of microRNA inhibitors. Silence, vol. 1, no. 1, p. 10. [CrossRef]

WANG, F.; FU, X.D.; ZHOU, Y. and ZHANG, Y. (2009). Down-regulation of the cyclin E1 oncogene expression by microRNA-16-1 induces cell cycle arrest in human cancer cells. BMB Report, vol. 42, no. 11, p. 725-730.

WILLENBROCK, H.; SALOMON, J.; SØKILDE, R.; BARKEN, K.B.; HANSEN, T.N.; NIELSEN, F.C.; MØLLER, S. and LITMAN, T. (2009). Quantitative miRNA expression analysis: Comparing microarrays with nextgeneration sequencing. RNA, vol. 15, no. 11, p. 2028-2034. [CrossRef]

ZHENG, L.; BAUMANN, U. and REYMOND, J.L. (2004). An efficient one-step site-directed and site-saturation mutagenesis protocol. Nucleic Acids Research, vol. 32, no. 14, p. e115. [CrossRef]

\section{How to cite this article:}

BI, Y.; ZHENG, X.; SHAO, C.; PAN, W.; JIANG, L. and OUYANG, H. (2011). Construction and application of a built-in dual luciferase reporter for microRNA functional analysis. Electronic Journal of Biotechnology, vol. 14, no. 2. http://dx.doi.org/10.2225/vol14-issue2-fulltext-9 